\title{
Paper
}

\section{Still Images of High Spatial Resolution Enable Short TV Viewing Distances}

\author{
Masaki Emoto ${ }^{\dagger}$ (member) and Masayuki Sugawara ${ }^{\dagger}$ (member)
}

\begin{abstract}
Preferred viewing distance (PVD), which is the viewing distance favored in the home viewing environment, is important for overall TV broadcasting system assessments to guarantee the image quality of TV programs. Previous studies have suggested that image size and resolution have little effect on the PVD. However, in future TV systems such as ultra-high-definition TVs (UHDTVs), which are expected to have a wide field of view (FOV) and high spatial resolution, program production methods may change depending on the specific values of these parameters, and viewer behaviors may change accordingly. Here we used a program consisting of still images to show that viewer behavior differs between two spatial resolutions. This result suggests that the conditions in previous studies discouraged viewers from finding an optimized PVD for each scene because there was little need and insufficient time to move spontaneously.
\end{abstract}

Keywords: TV viewing distance, ultra-high-definition TV, behavior, memory.

\section{Introduction}

Ultra-high-definition TV (UHDTV) systems, which have been proposed for future broadcasting systems ${ }^{1) 2}$, have a wide field of view (FOV) and enable viewers to feel as though they are in the displayed space ${ }^{3) 4}$. This sensation-often referred to as a sense of presence or of "being there" can be perceived by viewers watching TV images of sufficient quality. However, simply increasing the image size for a wide-FOV display causes concomitant enlargement of the pixel size, which viewers may perceive as image blur. This degrades the subjective quality and sharpness of the image. To prevent this degradation, a wide-FOV TV system requires high spatial resolution. Hence, the UHDTV system is designed to have a resolution of 4320 scan lines by 7680 horizontal pixels and is often called the "8k system" due to its horizontal pixel number.

To produce programs for TVs with a wide FOV and high spatial resolution, new camera techniques may replace conventional ones. In traditional TV program production, zoom-ins are frequently used to show the details of an object of interest because the spatial resolution of conventional TVs is low. Moreover, the camera must pursue a moving object to keep it

Received October 10, 2013; Revised November 7, 2013; Accepted December 19,2013

$\dagger$ NHK Science \& Technology Research Laboratories

(Tokyo, Japan) continuously in the TV frame; otherwise, the object quickly moves out of the frame if the camera orientation is fixed. A TV system with a wide FOV and high spatial resolution displays an improved ability to show the details of objects-an ability that reduces the demand for zoom-ins. In addition, given its wide FOV, such a system also exhibits an improved ability to show moving objects with less camera movement. In fact, excessive camera movement in producing wide-FOV TV programs can increase the likelihood of visually induced motion sickness in viewers ${ }^{5)}$. Avoiding excessive camera movement or changes in camera orientation (i.e., panning and tilting) is desirable for reducing the risk of motion sickness.

Fewer zoom-ins may also change viewer behavior. For example, we hypothesize that viewers may adjust their viewing distances when watching a TV with a wide FOV and high spatial resolution, despite the inconvenience of decreasing or increasing viewing distances to see the details or overview of images, respectively. This hypothesis assumes that the behavior of a TV viewer is analogous to that of a viewer in an art museum, because both may reflect the desire to view the whole and details of images almost simultaneously. Demonstrating that a TV with a wide FOV and high spatial resolution could induce such seamless viewing behavior would constitute evidence that the system is advantageous.

To test whether viewers would optimize their viewing distances according to the spatial resolution presented, 
and whether they would be more likely to change their viewing position when watching images of wide FOV and high spatial resolution than when watching HDTV images, we conducted an experiment to compare behaviors including viewers' viewing distances while viewers watched an identical TV program except spatial resolution by one of two TVs with different spatial resolutions. Our major interest was the difference in viewing distance when viewers watched these two TV monitors, because the TV with high spatial resolution might enable viewers to see from a shorter distance than that with low spatial resolution. Our minor interests were the differences in subjective evaluations of image quality and detail, as well as the participants' behavior and memory for the content after the experiment. The subjective evaluations were conducted to investigate the relationship between the viewing distance, resolution, subjective image quality, and subjective image detail. Furthermore, the participants' investigation of related topics after the experiment and their memory for the content 10 days later were measured to test the effects of image resolution on behavior and memory.

\section{TV Viewing Distances}

In designing a TV broadcasting system, engineers should carefully consider two TV viewing distances: the design viewing distance (DVD) and preferred viewing distance (PVD).

\subsection{Design viewing distance (DVD)}

The DVD is important in TV broadcasting system assessments such as the subjective evaluation of image quality and performance evaluation of audio-visual equipment used in the broadcasting studio. The DVD is included among the standard viewing conditions recommended by the International Telecommunication Union, Radiocommunication Sector (ITU-R) ${ }^{6}$, which are useful for comparing the experimental results of various laboratories. In HDTV assessments, the standard viewing distance is three times the image height $(3 \mathrm{H})$. Although the rationale for this standard is not necessarily clear, three lines of convergent evidence have been used.

1. The standard viewing distance can be determined as a result of a subjective experiment ${ }^{7}$.

2. The subjective image quality corresponding to that of the HDTV can be defined as a function of the viewing distance ${ }^{8}$.

3 . The standard viewing distance can be defined as the distance where viewers with a visual acuity (VA) of
1.0 cannot resolve or perceive the scan lines or pixel structure on the display surface.

The viewing distance for UHDTV systems is not yet standardized. However, the third alternative above could be adopted simply based on a geometrical calculation without having to perform systematic subjective experiments. In this case, the standard viewing distance would be 0.75 times the image height $(0.75 \mathrm{H})$. Studies of UHDTV systems based on this standard have been conducted on the sense of presence ${ }^{4)}$ and visually-induced motion sickness ${ }^{5)}$.

\subsection{Preferred viewing distance (PVD)}

The PVD, which is defined as the favored viewing distance in the home viewing environment ${ }^{7 / 9) 10}$, depends on the screen size or image height. When the PVD is represented as the ratio to the image height, it decreases as the image height increases. The PVD differs between still and moving images ${ }^{10)}$. These results were obtained and recommended for standard definition TV (SDTV) and HDTV ${ }^{9)}$. The PVD is important in overall TV broadcasting system assessments such as subjective evaluation of the image quality at the end of the TV program distribution chain. However, the PVD for UHDTV systems is not yet standardized because such systems are still under development.

Previous studies have suggested that image size and resolution have little effect on the PVD ${ }^{10) 11)}$. However, this appears to be inconsistent with the ITU-R BT.1127 ${ }^{8)}$, which defines TV image quality as a function of the relative viewing distance, and suggests that image resolution affects the image quality and viewing distance. In the studies cited above, there was only one PVD because viewers moved their chair to a preferred viewing position. If viewers could move freely to their preferred viewing position for each object viewed (e.g., by standing, which enables free excursion), the PVDs might differ from those of previous studies. This is especially true for moving images or fast changes of scene, which discourage viewers from changing their PVD because of inadequate time to move to a preferred viewing position.

In the present study, we measured dynamic changes in the PVD by giving viewers enough time to move to their preferred viewing position with long-lasting still images and a narrative that prompted them to attend to a certain part of the still images. We also tried to verify the previous result that image size and resolution have little effect on the PVD by using two LCDs that could present images that were identical except for the spatial resolution. 


\section{Methods}

The experiment was conducted according to the following procedure. The binocular VA of participants was measured after they provided signed informed consent. Participants were first shown the introduction to a TV program by using a HDTV monitor.

Next, two liquid crystal displays (LCDs) differing in spatial resolution (4k or HDTV) were used to present the same main TV program, which lasted $430 \mathrm{~s}$ and consisted of a series of still images accompanied by narration. Ideally, we would have used a UHDTV (8k) LCD as the high spatial resolution LCD instead of a $4 \mathrm{k}$ LCD. However, because 8k LCDs are not yet available, we used the $4 \mathrm{k}$ LCD to investigate the effects of high spatial resolution.

The main program, called the "Miracle Spaulding Collection," displayed "Ukiyoe" (color prints of everyday life in the Edo period in Japan) on a 4k or a HDTV LCD with a diagonal length of 56 in. The pictures in the program were all still images without zoom-ins. The narrator prompted the participants to attend to a number of topics represented by the still images. We down-sampled $4 \mathrm{k}$ images into HDTV images using Wavelet transform ${ }^{12)}$.

The experiment was based on a randomized, doubleblind, matched-pairs design. Seventeen pairs of participants were matched in terms of sex, age, and binocular VA. A single monitor resolution was assigned at random to a participant, who was unaware that two monitor resolutions were used in the experiment. The experimenter was also unaware of the resolution assignment, which was performed by a third person. Each viewer watched the program once on one of the LCDs, and adjusted his/her viewing distance freely. The viewing positions were recorded as a function of time. We defined the viewing distance as the length of perpendicular line to display surface from viewer.

We obtained approval from the Ethics Committee on Human Research of the Japan Broadcasting Corporation (NHK) Science and Technology Research Laboratories.

\subsection{Participants}

Sixty-eight healthy adults were enrolled and 34 participants were grouped into 17 pairs, each of which was matched on sex, age, and binocular VA (1 male and 16 female pairs; mean age, 31.5 years; range, 26-34; mean VA, 1.50). The participants were instructed to watch TV standing up and to select their viewing position freely by moving left, right, forward, and backward. The participants put on head gear with an upward-facing LED that was filmed by an overhead ceiling camera with a fish-eye lens to record the participants' excursions. When viewing of the program began, the participants' viewing distance was $53 \mathrm{~cm}$, which was 0.75 times the display height.

The distance between the display surface and the LED was about $63 \mathrm{~cm}$, including the viewing distance $(53 \mathrm{~cm})$ and the distance from the eyes to the LED atop the head $(10 \mathrm{~cm})$. This short initial viewing distance was chosen based on the result of a preliminary experiment, which showed that an ordinary initial viewing distance such as $3 \mathrm{H}$ tended to prevent participants from approaching the display and noticing that it had a high spatial resolution that would enable them to watch it at a shorter distance. After adopting an initial viewing distance of $0.75 \mathrm{H}$, most participants selected their viewing distance more freely.

\subsection{Subjective Evaluation}

Subjective evaluations of image quality and image detail were conducted after the participants viewed the main program. The participants recorded their ratings on two $10-\mathrm{cm}$ visual analogue scales (VAS) ${ }^{13) 14)}$ for the perceived image quality and degree of image detail. The subjective assessment was conducted in conformity with the ITU-R recommendations ${ }^{6) 9)}$ except viewing distance. Accordingly, the environmental illuminance on the screen was approximately $30 \mathrm{~lx}$ with D65 background. The height from floor to center of LCD monitors were $140 \mathrm{~cm}$. The participants were non-expert.

\subsection{Questionnaire}

In a questionnaire given 10 days after the viewing of the program, the participants were asked whether they had investigated related topics (e.g., via the Internet) after the experiment. The participants were also tested on their memory for the content. To assess recognition, the questionnaire sheet also included 63 two-alternative choices, each of which consisted of a target and a distractor. Fifty of the choices involved words, and 13 involved pictures. One of the alternatives (target) had been included in the experiment, but the other had not (distractor). The participants were instructed to check the alternative that they believed had been presented in the experiment.

\subsection{Apparatus}

The 4k LCD (Chi Mei Optoelectronics V562D1) had a spatial resolution of 2160 scan lines by 3840 horizontal pixels. The HDTV LCD (Sharp Aquos LC-57RX1W) had a spatial resolution of 1080 scan lines by 1920 horizontal 
pixels. Although the latter LCD had various controls (e.g., $120-\mathrm{Hz}$ drive, noise reduction) to improve the image quality, these functions were all disabled. Its luminance and color settings were also fully used to imitate the $4 \mathrm{k}$ LCD and minimize differences in the image characteristics between the two LCDs. The size of the room was $6.5 \mathrm{~m} \times 11.5 \mathrm{~m}$. The two LCDs were positioned side by side at one end of the room along the shorter wall.

The still images and narration were played back with a PC. The narration was presented by using a loudspeaker (Fostex 6301B). For each participant, one LCD was used and the other was turned off. Output images from the ceiling camera, which contained the participant's viewing position, were captured by a Hitachi ip-5000 image processing board to convert the fish-eye coordinates into rectangular coordinates. The data recorded on viewing position included the distance between the LCD surface and LED on the headgear. A single pixel of the ceiling camera corresponded to a maximum distance of $4 \mathrm{~cm}$.

\subsection{Data Analysis}

The average viewing distance was calculated for each participant as a function of time. The averaged and paired data for the $4 \mathrm{k}$ and HDTV LCD groups were analyzed by a paired t-test, and Cohen's d was calculated for the effect size. To investigate the temporal aspect of the viewing distances for the $4 \mathrm{k}$ and HDTV LCD resolutions, a cross-correlation analysis was performed between the two time-varying viewing distance curves.

Paper VAS ratings were measured with a ruler to obtain the distances from the origin, and the distance data were analyzed by a paired t-test to verify the rating differences between the two LCD resolutions.

The numbers of participants who reported investigating related topics after their experiment were counted, and McNemar's test was conducted to compare the numbers between the two LCD resolutions.

The number of correct choices in the recognition test was counted for each participant. The difference in this number between the two LCD resolutions was analyzed by a paired t-test.

\section{Results}

The viewing distances of participants who watched the 430-s TV program were shorter for the 4k LCD group than for the HDTV LCD group (Fig. 1). The difference between the viewing distances ranged from 20 to $50 \mathrm{~cm}$,

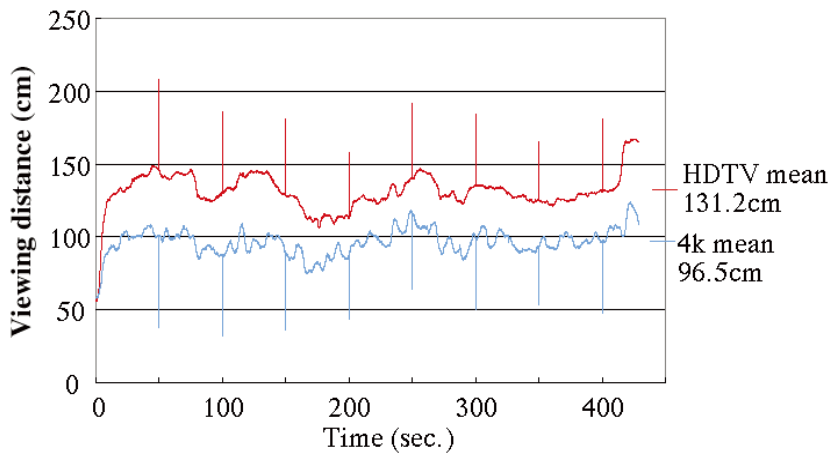

Fig.1 Averaged viewing distances for participants $(n=17)$ viewing a 430 -s TV program on a $4 \mathrm{k}$ and HDTV LCD. Error bars were added every $50 \mathrm{~s}$ to show the standard deviations. The viewing distance of the $4 \mathrm{k} \operatorname{LCD}(\mathrm{M}=96.49, \mathrm{SD}=29.52)$ was significantly shorter than that of the HDTV LCD ( $\mathrm{M}=$ $131.19, \mathrm{SD}=44.75, \mathrm{t}(16)=2.371, \mathrm{P}=0.031$, Cohen's $\mathrm{d}=$ 0.92). The temporal cross-correlation of the viewing distances for $4 \mathrm{k}$ and HDTV LCD was 0.782 . The two curves on the figure show local dips at the same periods in the program (i.e., around 80, 170, and $340 \mathrm{~s}$ ), representing the viewer's approaches to the LCD.

and the two curves were similar except for the mean value of the waveform (or the DC offset). A paired t-test showed that the viewing distance for the $4 \mathrm{k}$ LCD ( $M=$ 96.49 , SD = 29.52) was significantly shorter than that for the HDTV LCD $(\mathrm{M}=131.19, \mathrm{SD}=44.75, \mathrm{t}(16)=$ $2.371, \mathrm{P}=0.031$, Cohen's $\mathrm{d}=0.92$ ). This result indicates that spatial resolution is an important factor that affects the viewing distance. To conduct a temporal crosscorrelation analysis of the viewing distances for the two LCD resolutions, we subtracted the DC level from the two time-varying curves of viewing distance and calculated the cross-correlation coefficient and lag time. The lag time was zero, and the cross-correlation coefficient was 0.782 . This strong cross-correlation indicates that the viewing distances for the $4 \mathrm{k}$ and HDTV LCDs changed in parallel, and that the content of the program at each time point was an important factor affecting the viewing distance.

Two paired t-tests were conducted to assess differences in the subjective evaluation of image quality and image detail between the two LCD resolutions (Table 1). The results showed that the subjective evaluation of the image quality of the $4 \mathrm{k}$ LCD ( $\mathrm{M}=$ $87.53, \mathrm{SD}=12.17$ ) was not significantly different from that of the HDTV LCD $(\mathrm{M}=84.18, \mathrm{SD}=18.16, \mathrm{t}(16)=-$ $0.629, \mathrm{P}=0.538$, Cohen's $\mathrm{d}=0.22$ ). The results also showed that the subjective evaluation of the image detail of the $4 \mathrm{k}$ LCD ( $\mathrm{M}=87.82, \mathrm{SD}=14.14)$ was not significantly different from that of the HDTV LCD ( $\mathrm{M}=$ 77.35, $\mathrm{SD}=13.68, \mathrm{t}(16)=-2.078, \mathrm{P}=0.054$, Cohen's $\mathrm{d}=$ 
Table 1 Results of the subjective evaluations and recognitions.

\begin{tabular}{cccccc}
\hline \hline & & \multicolumn{2}{c}{$4 \mathrm{k}$} & \multicolumn{2}{c}{ HDTV } \\
\cline { 2 - 6 } & & Mean & SD & Mean & SD \\
\hline \multirow{2}{*}{$\begin{array}{l}\text { Subjective } \\
\text { evaluation }\end{array}$} & Quality & 87.53 & 12.17 & 84.18 & 18.16 \\
\cline { 2 - 6 } & Detail & 87.82 & 14.14 & 77.35 & 13.68 \\
\hline \multirow{2}{*}{ Recognition } & Words & 35.47 & 3.81 & 35.53 & 2.92 \\
\cline { 2 - 6 } & Pictures & 9.76 & 1.68 & 8.47 & 2.24 \\
\hline
\end{tabular}

0.75). Taking the result of the viewing distance into consideration, these results suggest that the participants avoided the degradation of image quality by controlling their viewing distance (i.e., longer viewing distances for the HDTV LCD than for the 4k LCD to avoid image blur). The shorter viewing distances for the $4 \mathrm{k}$ LCD in turn enabled the participants to perceive more detail in the images.

The paired t-test for the number of correct choices for word alternatives showed no significant difference between the HDTV $(\mathrm{M}=35.47, \mathrm{SD}=3.81)$ and $4 \mathrm{k} \mathrm{LCD}$ $(\mathrm{M}=35.53, \mathrm{SD}=2.92, \mathrm{t}(16)=-0.049, \mathrm{P}=0.961$, Cohen's $d=0.22$ ). The paired t-test for the number of correct choices for picture alternatives also showed no significant difference between the HDTV $(\mathrm{M}=8.47, \mathrm{SD}$ $=2.24)$ and $4 \mathrm{k} \operatorname{LCD}(\mathrm{M}=9.76, \mathrm{SD}=1.68, \mathrm{t}(16)=-1.67, \mathrm{P}$ $=0.11$, Cohen's $\mathrm{d}=0.66)$. Recognition rates were significantly beyond the chance levels of 25 for words (M $=35.47, \mathrm{SD}=3.81, \mathrm{t}(16)=11.33, \mathrm{P}<0.01$, for HDTV $\mathrm{M}$ $=35.53, \mathrm{SD}=2.92, \mathrm{t}(16)=14.88, \mathrm{P}<0.01$, for $4 \mathrm{k})$ and 6.5 for pictures $(\mathrm{M}=8.47, \mathrm{SD}=2.24, \mathrm{t}(16)=3.63, \mathrm{P}<$ 0.01 , for HDTV; $\mathrm{M}=9.76, \mathrm{SD}=1.68, \mathrm{t}(16)=8.02, \mathrm{P}<$ 0.01 , for $4 \mathrm{k}$ ). These results suggest that the participants successfully remembered the words and pictures included in the experiment. The moderate effect size for picture recognition suggests that if the difference in spatial resolution between the two LCDs was larger than in the present experiment (i.e., $8 \mathrm{k}$ instead of $4 \mathrm{k}$ spatial resolution), a significant difference in recognition might be detected.

The number of participants who reported investigating related topics after the experiment was zero after viewing the HDTV LCD and two after viewing the $4 \mathrm{k}$ LCD. However, this difference was not statistically significant by McNemar's test $(\mathrm{P}=0.480)$.

\section{Discussion}

We found a significant difference in mean viewing distance between two LCDs with different spatial resolutions (4k and HDTV), and the viewing distances for each changed in parallel across time. We also compared the shortest and longest viewing distances between the two spatial resolutions. To avoid the effect of initial viewing distance, the shortest and longest viewing distances were determined for each participant in the 30-400 s period. Only the shortest viewing distance of the $4 \mathrm{k} \operatorname{LCD}(\mathrm{M}=63.49, \mathrm{SD}=21.16)$ was significantly shorter than that of the HDTV LCD $(\mathrm{M}=$ 92.04, $\mathrm{SD}=40.35$; paired t-test, $\mathrm{t}(16)=2.33, \mathrm{P}=0.033$, Cohen's $d=0.89$ ), whereas the longest viewing distances were not significantly different. This shows that the higher spatial resolution prompted viewers to view the LCD at a shorter viewing distance. Taking the results of the subjective evaluations into consideration, this finding suggests again that participants can avoid the degradation of image quality by controlling their viewing distance to perceive the details of images presented on the LCD with high spatial resolution.

The curves in Fig. 1 also show a number of local dips at the same time points during the program (i.e., around 80,170 , and $340 \mathrm{~s}$ ), representing viewers' temporary approaches toward the LCD. The appearance of Japanese characters on the screen ( $80 \mathrm{~s})$ first prompted the participants to pay close attention. Subsequently, the narrator described sophisticated techniques used to print the receding hairline of a woman ( 170 s; Fig. 2), and the participants were then prompted to answer a quiz to find three differences between two versions of an Ukiyoe print $(\sim 340 \mathrm{~s})$. The spatial frequencies of the Japanese characters, receding hairline, and details of the prints were high, and viewers needed to approach the LCD to view the details or components with high spatial frequencies. Fig. 3 shows two-dimensional DFT (Discrete Fourier Transformation) corresponding to Fig.

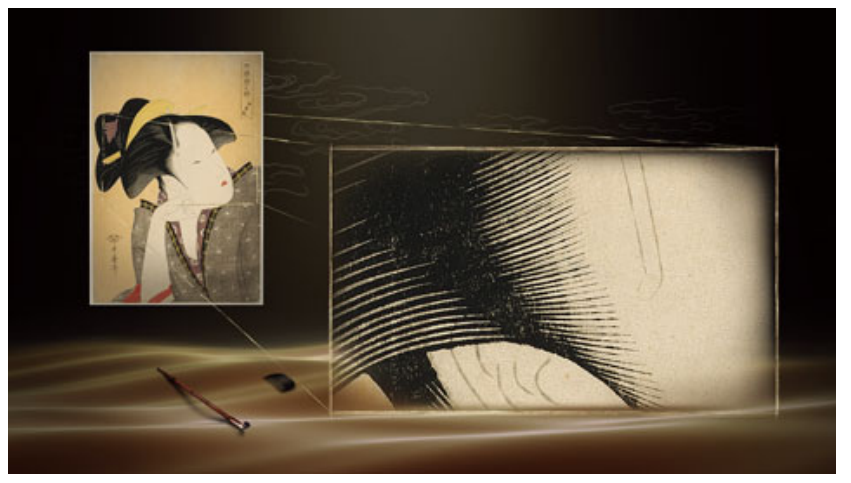

Fig.2 Image presented around $170 \mathrm{~s}$. into the program and a magnified image of the region to which attention was directed. The participants were prompted to pay attention to the receding hairline of the woman. 

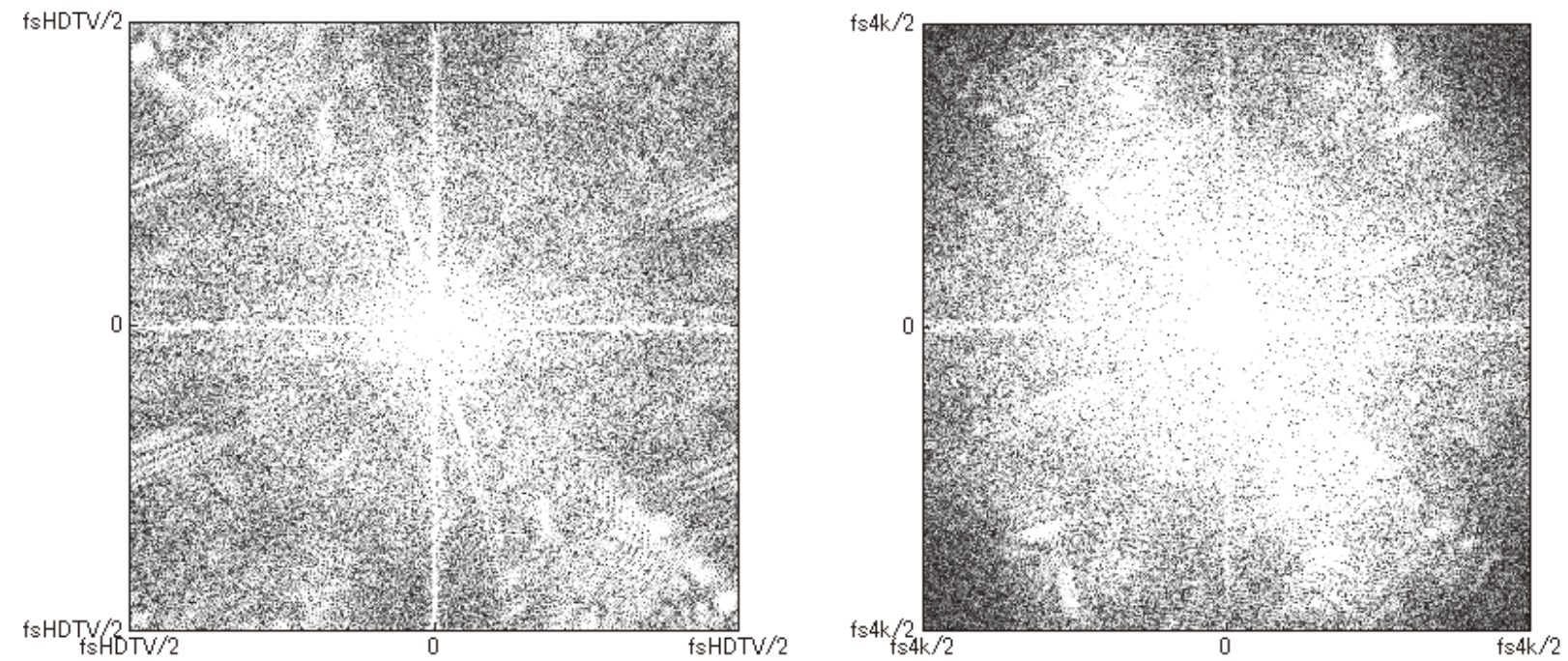

Fig.3 Two-dimensional DFT (Discrete Fourier transformation) corresponding to fig. 2. Left panel shows power spectrum of HDTV image and right panel shows $4 \mathrm{k}$ one. FsHDTV and fs4k indicates sampling frequency of each image respectively. 4k image had richer power spectrum in high spatial frequency than HDTV.

2. The power spectrum of each image showed that $4 \mathrm{k}$ image had richer power spectrum in high spatial frequency than HDTV. This suggests that the viewing distance was related to the spatial frequency of the subject of focus presented on the TV monitor.

In previous studies on the PVD ${ }^{10)}{ }^{11)}$, researchers have concluded that there is a single PVD for a given TV system. This might indicate that viewers decide on a single PVD for watching TV at home in a relaxed and passive manner if there is no need for spontaneous movement. However, this lack of movement would discourage the discovery of an optimized PVD for each scene with various spatial frequency components, much as would moving images where there is insufficient time to adjust the PVD.

\section{Conclusion}

We conducted an experiment to compare behaviors including viewers' viewing distances while viewers watched an identical TV program except spatial resolution by one of two TVs with different spatial resolutions. The present results show that the spatial resolution of the presented objects of interest affects the viewing distance (i.e., the viewing distance decreases when TV images are presented with high spatial resolution). They also show that participants can adjust and optimize their viewing distances actively when they are sufficiently motivated (i.e., by narration in the present study) and presented with still images for an adequate amount of time. These results successfully verify our hypothesis that the viewing distance changes for TV programs with a wide FOV and high spatial resolution. We compared viewer behaviors in response to $4 \mathrm{k}$ and HDTV resolutions and detected a significant difference in viewing distance. If the difference in spatial resolution were greater than in the present study (i.e., $8 \mathrm{k}$ instead of $4 \mathrm{k}$ ), we would expect to find a greater difference in the viewing distances. Further comparisons between $8 \mathrm{k}$ and HDTV resolutions in the future will be required because the $8 \mathrm{k}$ UHDTV system is proposed as a future broadcasting system.

\section{References}

1) M. Kanazawa, K. Hamada, I. Kondoh, F. Okano, Y. Haino, M. Sato and K. Doi: "An ultrahigh-definition display using the pixel-offset method", SID, 12, pp.93-103 (2004)

2) M. Sugawara, M. Kanazawa, K. Mitani, H. Shimamoto, T. Yamashita and F. Okano: "Ultrahigh-definition video system with 4000 scanning lines", SMPTE Motion Imag., 112, pp.339-346 (2003)

3) T. Hatada, H. Sakata and H. Kusaka: "Psychophysical analysis of the 'sensation of reality' induced by a visual wide-field display", SMPTE J. 89, pp.560-569 (1980)

4) M. Emoto, K. Masaoka, M. Sugawara and Y. Nojiri: "The viewing angle dependency in the presence of wide field image viewing and its relationship to the evaluation indices", Displays, 27, pp.80-89 (2006)

5) M. Emoto, M. Sugawara and Y. Nojiri: "Viewing angle dependency of visually-induced motion sickness in viewing wide-field images by subjective and autonomic nervous indices", Displays, 29, pp.90-99 (2008)

6) International Telecommunication Union, Radiocommunication Sector (ITU-R). Subjective assessment methods for image quality in high-definition television. Recommendation ITU-R BT. 710-4 (1998)

7) T. Fujio: "High-definition television systems", Proc IEEE, 73, pp.646-655 (1985)

8) ITU-R. Relative quality requirements of television broadcast systems. Recommendation ITU-R BT. 1127 (1994)

9) ITU-R. Methodology for the subjective assessment of the quality of television pictures. Recommendation ITU-R BT. 500-13 (2012)

10) M. Ardito: "Studies of the influence of display size and picture brightness on the preferred viewing distance for HDTV programs", SMPTE J. 103, pp.517-522 (1994) 
11) A.M. Lund: "The influence of video image size and resolution on viewing-distance preferences", SMPTE J. 102, pp.406-415 (1993)

12) T. Kuge: "Wavelet picture coding and its several problems of the application to the interlace HDTV and the ultra-high definition images", IEEE 2002 International conference on image processing (ICIP2002) WA-P2.1 (2002)

13) M.H. S. Hayes and D.G. Patterson: "Experimental development of the graphic rating scale", Psychology Bulletin, 18, pp.98-99 (1921)

14) M.J. Freyd: "The graphic rating scale", Educational Psychol. 14, p.83 (1923)

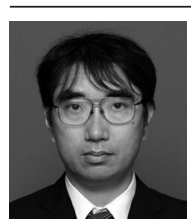

Masaki Emoto received his B.S. and M.S degrees in electronic engineering and his $\mathrm{PhD}$ degree in human and environmental studies from Kyoto University in 1986, 1988, and 2003, respectively. He has been working on future television systems and human science, especially human visual sysetm, at NHK Science and Technical Research Laboratories. His research interests include future television systems incorporating desirable effects such as a heightened sensation of presence, and eliminating undesirable side effects to the viewer such as photosensitive response, visually induced motion sickness, and visual fatigue.

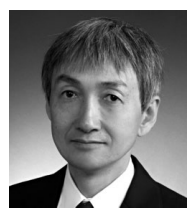

Masayuki Sugawara received the B.S. and M.S. degrees in electric communication engineering, $\mathrm{Ph} . \mathrm{D}$. degree in electronic engineering from Tohoku University, Sendai, Japan. He joined NHK (Japan Broadcasting Corporation), Tokyo, Japan, in 1983. Since 1987, he has been researching solid-state image sensor and HDTV camera at Science and Technology Research Laboratories. He was an associate professor at the University of Electro-Communications in Tokyo from 2000 until 2004. He has been attending ITU-R SG6 meeting since 2004 and been active in the working parties that deal with programme production. At present, he is Head of Advanced Television Systems Research Division at NHK STRL and is engaged in the research of ultra high definition TV system. He is a member of SMPTE, IEEE, SID, IEICE and ITE. 